\title{
विकासात्मक पत्रकारिता संबंधित खबरों का अवलोकन एवं अध्ययनः प्रिन्ट मीडिया के विषेष संदर्भ में
}

दीपक कुमार

सारांश

विकास एक ऐसी सतत् गतिमान प्रक्रिया है, जिसका लाभ जन-जन को मिलें। प्रत्येक विकास कार्यक्रम का उद्देश्य है व्यक्तिगत, सामाजिक और राष्ट्रीय जीवन का हित व कल्याण। विकास का अर्थ है सामाजिक, आर्थिक, सांस्कृतिक, शैक्षणिक, प्रौद्योगिकी विकास द्वारा सामाजिक व्यवस्था में सुधार, सामाजिक-राष्ट्रीय सुरक्षा में सुधार। समुचे देश में सुख समृद्धि में वृद्धि। हर तरह के शोषण, भेदभाव, पक्षपात, अन्यान्य, विषमता जैसी अमानवीय बुराईयों की समात्ति। शिक्षा एवं ज्ञान का फैलाव, स्वास्थ्य एवं जीवन की आवश्यक सुविधाओं का विकास। सबको समान अवसर और लोक कल्याणकारी राज्य एवं समाज की स्थापना की सतत कोशिश। नैतिक, मानसिक, आध्यात्मिक विकास के साथ-साथ जीवन मूल्यों को जीवन में आचरण द्वारा प्रतिष्ठापित करने की कोशिश। इसके लिए समाज में पत्रकारिता की विषेष भूमिका है, विकास की बात विकासत्मक पत्रकारिता से बेहतर और कौन कर सकता है। अतः प्रस्तुत शोध में भारत में विकासात्मक पत्रकारिता की भूमिका का अध्ययन एवं समाचार पत्र-एवं पत्रिकाओं में विकास संबंधित खबरों का अवलोकन किया जा रहा है।

कूट शब्द. विकास, विकासात्मक पत्रकारिता, प्रिन्ट मीडिया, खबरों का अवलोकन। 


\section{अध्ययन की भूमिका}

भारत विकासशील देश है, किसी भी विकासशील देश के लिए विकास का कितना महत्व है यह सभी जानते है। भारत विश्व की सबसे बड़ी अर्थशक्तियों में से एक है। जबकि चीन के बाद दूसरी सबसे तेजी से विकास करने वाला देश भारत ही है। लेकिन क्या यह सच्चाई है? क्या वास्तव में भारत प्रगति कर रहा है? महंगाई, भूख, अशिक्षा, कुपोषण, बेरोजगारी भ्रप्टाचार की बढ़ती समस्या को दर किनार कर क्या हम भारत को विकसित देश के रुप में देखना पसंद करेगें।

क्या वास्तव में विकास की यही परिभाषा है? केवल आर्थिक विकास को ही हम विकास मानें! राजनीतिक, सामाजिक, सांस्कृतिक व आध्यात्मिक मूल्यों का विकास क्या कोई मायने नहीं रखता।

लोग गांव छोड़कर शहर की ओर पलायन कर रहें है कई किसान आत्महत्या कर रहे है, खेती योग्य भूमि सिमटती जा रही है। गरीब और अधिक गरीब होते जा रहे है। गांवों में रोटी, कपड़ा, मकान तथा स्वास्थ्य और शिक्षा की समस्या बनी हुई है। क्या इसे ही विकास कहेंगे?

पत्रकारिता जगत में हत्या लूट व मनोरंजक व राजनीतिक खबरों का भरमार होता है लेकिन विकास संबंधी खबरें न के बराबर होती है। कुछ खबरें होती भी है, तो इसे निरंतरता नहीं दिया जाता।

इस शोध में हम जानेंगे कि वास्तव में विकास क्या है। समाचार पत्र-पत्रिकाओं में इसे कितना महत्व दिया जाता है। प्रकाशित खबरों का उस क्षेत्र के विकास में क्या प्रभाव पड़ता है।

शोध उद्देश्य

1. भारत में विकासात्मक पत्रकारिता की भूमिका का अध्ययन।
2. समाचार पत्र एवं पत्रिकाओं में विकास संबंधित खबरों का अवलोकन करना।

\section{साहित्य सर्वेक्षण}

विकासात्मक पत्रकारिता पर कई शोध किए गए हैं। जिसमें से एवरीबडी लब्ज ए गुड ड्रोंट, पी साईनाथ के अध्ययन को शोधार्थी द्वारा साहित्य सर्वेक्षण के रूप में प्रस्तुत किया गया है।एवरीबडी लब्ज ए गुड ड्रोंट, पी. साईनाथ (1996) - इसके जरिए पी. साईनाथ ने भारत के सबसे गरीब जिलों में रहने वाले लोगों की व्यथा को उभारने की कोशिश की हैं इसमें उन्होंने बताया कि कैसे एक गरीब व्यक्ति दिन का 10 रूपये कमाने के लिए कई मीलों का सफर पैदल तय करता है। इसमें उन्होंने भारत के आठ करोड़ गरीब और आदिवासियों की कहानियां बताई हैं।पी. साईनाथ के अध्ययन से विकासात्मक पत्रकारिता के विभिन्न आयामों को समझने का अवसर प्राप्त हुआ, जिसका उपयोग शोधकर्त्ता ने प्रस्तुत शोध में किया है।

\section{शोध विधि}

प्रस्तुत शोध के लिए समाचार पत्र-पत्रिकाओं का अन्तःवस्तु अवलोकन किया गया है। इसके लिए देहरादून एवं दिल्ली से प्रकाशित होने वाले पांच समाचार पत्र एवं पांच पत्रिकाओं का अध्ययन किया गया। यह शोध मुख्यतः विश्लेषणात्मक है। इस कार्य में उपलब्ध पुस्तकों, समाचार पत्र-पत्रिकाओं व इंटरनेट का सहारा लिया गया है।

\section{शोधविवेचना एवं व्याख्या}

विकास का अर्थ

विकास का उद्देश्य वैज्ञानिक, सामाजिक और आर्थिक उपलब्धि के साथ मानवीय अवधारणाओं, मूल्यों, स्वास्थ्य, सुरक्षा और शासन में सहभागिता है। द्वितीय विश्वयुद्ध के बाद में शिक्षा, स्वास्थ्य, सामाजिक और सांस्कृतिक पक्षों की प्रगति भी उसमें जुड़ी। अब तो समग्र विकास पर जोर दिया जा रहा है। 
अपने यहाँ भौतिक उत्कर्ष के साथ-साथ मानवीय मूल्यों का उत्कर्ष भी विकास का एक आवश्यक अंग माना गया है। अपने यहाँ व्यक्ति समाज की इकाई है। व्यक्तिगत जीवन की समुन्नति ही सामाजिक जीवन की आधारशीला है। ब्रह्मचर्य, गृहस्थ, वानप्रस्थ और संत्यास के इन चारों अवस्थाओं में धर्म, अर्थ, काम व मोक्ष की प्रात्ति से विकास पूर्णता को प्राप्त हो सकता है।

\section{विषेषज्ञ के अनुसार विकास की परिभाषा}

इन्वर्ट- "विकास का वास्तविक अर्थ तकनीकी या राष्ट्रीय उत्पादकता में वृद्धि नहीं है बल्कि ज्ञान एवं चेतना के उस विकास से है जिसके द्वारा वह सहभागी बनता है। मैन, मशीन, मनी और मीडिया ये चार आधारभूत तत्त्व हैं जिन पर विकास आधारित है।"

\section{विकासात्मक पत्रकारिता}

विकास संबंधी खबरों को प्रकाशित करने वाला पत्रकारिता विकासात्मक पत्रकारिता के अंतर्गत आता है। आर्थिक, सामाजिक, सांस्कृतिक क्षेत्रों के बारे में रचनात्मक लेखन को विकास पत्रकारिता के रूप में माना गया है। पत्रकार का मुख्य कार्य अपने पाठकों को तथ्यों की सूचना देना हैं, जहाँ सम्भव हो वहां निष्कर्ष भी दिया जा सकता है। अपराध तथा राजनीतिक संवाददाताओं का यह मुख्य कार्य है। तीसरा एक मुख्य दायित्व प्रसार का है। आर्थिक-सामाजिक जीवन के बारे में तथ्यों का प्रस्तुतीकरण ही पर्याप्त नहीं, लेकिन उनका प्रसार भी आवश्यक है। गम्भीर विकासात्मक समस्याओं से पाठकों को अवगत कराना भी आवश्यक है। पाठक को सोचने के लिए विवश कर इस प्रकार का लेखन सम्भावित समाधानों की ओर भी संकेत करता है। विकासात्मक लेखन में शोध का भी पर्याप्त महत्व है।

शुद्ध विकासात्मक लेखन के क्षेत्रों में रविवार के तत्कालीन विशेषज्ञ संवाददाता राजीव शुक्ल ने 18 भागों में विभक्त किया है - उद्योग, कृषि, शिक्षा और साक्षरता, आर्थिक गतिविधियां, नीति और योजना,
परिवहन, संचार, जनमाध्यम, ऊर्जा और ईंधन, श्रम व श्रमिक कलयाण, रोजगार, विज्ञान और तकनीक, रक्षा, रक्षा अनुसन्धान औरउत्पाद तकनीक, परिवार नियोजन, स्वास्थ्य चिकित्सा सुविधाएँ, शहरी विकास, ग्रामीण विकास, निर्माण और आवास, पर्यावरण और प्रदूषण।

आर्थिक विकास निवेश, उत्पादन और उपभोग में वृद्धि का पर्याय है। इनमें विसंगति ही दीख पड़ती है। जमीन का किसान हित में न्यायसंगत बँटवारा, जीवन के लिए आवश्यक खाद्य पदार्थों का उत्पादन, स्वास्थ्य के साधनों में विसंगति है, जिससे विकास की गति धीमी है। मुट्ठीभर लोग अधिकांश लोगों की गरीबी, बेरोजगारी, बदहाली पर विकसित हो रहे हैं। विकास एकपक्षीय नहीं अपितु बहुपक्षीय होता है। सभी क्षेत्रों में सभी अंगों को गतिशील बनाना विकास लाना है। राष्ट्र के संस्थानों की व्यवस्था जैसे वित्त, उद्योग बैंक, तकनीकी व्यवस्था को सुदृढ़ बनाना विकास का लक्ष्य है।

यहाँ विश्व बैंक की 1994 में प्रकाशित विश्व विकास रिपोर्ट का जिक्र करना प्रासंगिक होगा जिसमें विकास के लिए बुनियादी ढाँचे को मुख्य विषय बनाया गया है। विश्व बैंक के अनुसार आधारभूत आर्थिक ढाँचे में जनोपयोगी सेवाएँ जैसे बिजली, दूरसंचार, पाइप लाइनों से जलापूर्ति, स्वच्छता और जल-मल निकासी, ठोस कूड़े का संग्रह तथा निपटान, गैस की सप्लाई, सड़कों, बाँधों, नहरों आदि से संबंधित लोक निर्माण कार्य, सिंचाई और वर्षा के जल की निकासी, हवाई अडुों, बंदरगाहों, जल मार्गो, शहरी परिवहन तथा शहरों के बीच यातायात जैसे परिवहन सेवाएँ आती हैं। दरअसल आधारभूत ढाँचा एक ऐसा विस्तृत अर्थ वाला शब्द है जिसके अन्तर्गत कई तरह की गतिविधियाँ आ जाती हैं। सुप्रसिद्ध विकास अर्थशास्तियों पॉल रोजन्स्टीन रॉडन और राग्नर नकर्से ने इन गतिविधियों को 
"सामाजिक पूँजी" नाम दिया है। आज सभी विकास योजनाओं के बावजूद गरीबी अमीरी का अन्तर बढ़ा है।

\section{विकास पत्रकारिता का उद्देण्य}

विकास एक ऐसी सतत् गतिमान प्रक्रिया है, जिसका लाभ जन-जन को मिलें। प्रत्येक विकास कार्यक्रम का उद्देश्य है व्यक्तिगत, सामाजिक और राश्ट्रीय जीवन का हित व कल्याण। विकास का अर्थ है सामाजिक, आर्थिक, सांस्कृतिक, शैक्षणिक, प्रौद्योगिकी विकास द्वारा सामाजिक व्यवस्था में सुधार, सामाजिक-राष्ट्रीय सुरक्षा में सुधार। समुचे देश में सुख समृद्धि में वृद्धि। हर तरह के शोषण, भेदभाव, पक्षपात, अन्यान्य, विषमता जैसी अमानवीय बुराईयों की समात्ति। शिक्षा एवं ज्ञान का फैलाव, स्वास्थ्य एवं जीवन की आवश्यकसुविधाओं का विकास। सबको समान अवसर और लोक कल्याणकारी राज्य एवं समाज की स्थापना की सतत कोशिश। नैतिक, मानसिक, आध्यात्मिक विकास के साथ-साथ जीवन मूल्यों को जीवन में आचरण द्वारा प्रतिष्ठापित करने की कोशिश। पिछड़ापन बर्बरता, संकुचितता, निरंकुशता, भाग्यवाद का क्रमशः अंत। निर्धनता, विपन्नता, असहाय अवस्था, निष्क्रियता, हीनता, दिशाहीनता और अज्ञान की जकड़न से निकलकर क्रमशः संपन्नता आत्मनिर्भरता, स्वाभिमानपूर्ण, व्यक्तिगत,सामाजिक-राष्ट्रीय जीवन की स्थापना और इंसानियत की प्रतिष्ठा की ओर कदम बढ़ाना ही विकास है।भौतिक उत्कर्ष के साथ-साथ मानवीय मूल्यों का उत्कर्ष भी विकास का आवश्यक अंग है। चूंकि व्यक्ति समाज की इकाई है, इसलिए व्यक्तिगत जीवन समुन्नति ही सामाजिक जीवन की आधारशीला है। वैसे प्रत्येक देश और समाज में विकास एवं प्रगति की अपनी-अपनी धारणायें होती हैं। अपनी-अपनी जरूरतों, महत्वकांक्षाओं,तमन्नाएं और वर्चस्व स्थापित करने की इच्छाएं भी होती हैं जिनके कारण टकराव, युद्ध एवं विनाश के हालात बनते हैं लेकिन मानव स्वाभाव है कि
वह फिर विकास की ओर बढ़ता है। विकास प्रक्रिया का इंसानी और सामाजिक जिंदगी से सीधा वास्ता है। इसमें पत्रकारिता का योगदान सहायक होता है।

\section{विकासात्मक पत्रकारिता का वर्तमान स्वरुप}

भारतीय विकासात्मक पत्रकारिता अपने नये रुप में इस दषक में सामने आई है क्यांकि नया दौर विकास को ही समर्पित है भारत जैसे विकासषील देष के लिए इसका महत्व और भी अधिक बढ़ जाता है वर्तमान में विकास पत्रकारिता का महत्व उसी प्रकार बढ़ा है जैसे किसी जंगल को बचाए रखने के लिए पेडो का महत्व।

अतः वर्तमान में विकास पत्रकारिता को समझने के लिए साहित्य सर्वेक्षण से अच्छा कोई माघ्यम नहीं हो सकता। इसके लिए मैने पांच सामाचार पत्र और पांच मैग्जीन का अन्तःवस्तु विष्लेषण करने का निर्णय लिया जिससे यह बिल्कुल स्पष्ट हो जाए कि वर्तमान समय में विकासात्मक पत्रकारिता किस दौर से गुजर रहा है।

\section{अन्तःवस्तु विष्लेषण - पांच सामाचार पत्रों का अवलोकन}

विकास संबंधी खबरों का सर्वेक्षण के लिए पूरे एक महीने के पांच सामाचार पत्रों का अवलोकन किया गया। इसके लिए साल के महत्वपूर्ण महीने मार्च को चुना गया। क्योंकि सरकारी कैलेण्डर के अनुसार मार्च में ही पूरे साल के विकास कार्यो का विवरण दिया जाता है। उपरोक्त विकासात्मक पत्रकारिता की विषेषताओं को आधार बनाकर शोधार्थी ने शोध में शामिल समाचार पत्रों एवं पत्रिकाओं के विषयवस्तु का अन्तःविष्लेषण किया है। 


\begin{tabular}{ll}
\hline सामाचार-पत्र & विकास संबंधी खबरें \\
\hline अमर उजाला & 356 \\
\hline हिन्दुस्तान & 238 \\
\hline दैनिक जागरण & 270 \\
\hline द हिन्दु & 381 \\
\hline इंडियन एक्सप्रेस & 305 \\
\hline समयावधि- 1 मार्च 2012 से 31 मार्च 2012 तक \\
\hline
\end{tabular}

दैनिक जागरण

हिन्दी सामाचार पत्रों में दैनिक जागरण का विस्तार भारत के सबसे अधिक है इसके बावजूद स्थानीय व राजनीतिक खबरें इसमें प्रमुखता से दी जाती है । अवलोकन से यह ज्ञात होता है कि स्थानीय विकास संबंधी खबरों को तो दिया जाता है परन्तु राष्ट्रीय विकास की खबरें कम होती है।

शीर्षक - गांव में तेजी से कराए जा रहे निर्माण, उद्योगों के लिए दोहरा आघात, हरित क्रांति को शाबाशी दी तो गांव को भी कुछ-कुछ, विकास कार्या के लिए मिले दो और साल आदि शीर्षक से कई खबरें प्रकाशित हुई। पूरे मार्च महीने में कुल 270 खबरों का प्रकाशन किया गया।

\section{अमर उजाला}

उत्तर भारत में तेजी से विकास करने वाले अमर उजाला में विकास संबंधित खबरों की प्राथमिकता दी जाती है। मार्च महीन के अवलोकन में विकास संबिधित कुल 356 खबरों का प्रकाशन किया गया। जो हिन्दी सामाचार पत्रों में सबसे अधिक है।

शीर्षक:- विकास को तरस रहा श्यामपुर गांव, सिंचाई विभाग की सुस्ती स्कूलों पर भारी, खुद गंदगी से घिरे, कैसे साफ रखेंगे शहर आदि शीर्षक पर विकास की अच्छी कवरेज की गई है। " एक प्रतिभाशाली गरीब से सीधी बात" यशवंत व्यास जी का विशेष संपादकीय गरीबी पर लाईव रिर्पोटिग है।
हिन्दुस्तान

हिन्दुस्तान सामाचार पत्र स्थानीय विकास संबंधी खबरों को प्रमुखता से देता है। मार्च 2012 में गरीबी पर इसके कुछ संपादिय विशेष रहे। गरीबी के आंकड़ों की दरिद्रता में गरीबों के मुल्यांकन पर विभिन्न संस्थाओं के आंकड़ों को दर्शाया गया है। मार्च 2012 में कुल 238 खबरों का प्रकाशन इस पत्र ने किया।

द हिन्दु

अंग्रेजी के प्रमुख सामाचार पत्र विकासात्मक पत्रकारिता का अच्छा उदाहरण है। इसमें विकास की खबरें विस्तृत रुप से दी जाती है, साथ ही उसे निरंतरता भी दिया जाता है। मार्च 2012 में इसमें सबसे अधिक 381 खबरों का प्रकाशन किया गया।

विकासात्मक पत्रकारिता का यह आदर्श रुप प्रस्तुत करता है क्यांकि इसमें गांव से लेकर शहर तक के विकास के सभी आयामों को दिखाने की कोशिश की जाती है।

\section{इंडियन एक्सप्रेस}

अंग्रेजी पत्रकारिता में तेजी से विकास करता हुआ यह पत्र बड़े शहरों में काफी लोकप्रिय है। इसमें भी मार्च 2012 के दौरान विकास की अच्छी कवरेज देखने को मिली। गांव के लाइव रिपोर्टिंग के भी कई उदाहरण इसमें मिले। इसके ओपेड पेज खासकर विकास को ही समर्पित है। कुल 305 खबरों का प्रकाशन मार्च महीने में किया गया।

सारणी व्याख्याः- हिन्दी सामाचार पत्रों में अमर उजाला विकासात्मक खबरें पर अच्छा कवरेज करता है। इसका फोलोअप भी और अखबार की तुलना में बेहतर है। जबकी अंग्रेजी अखबारों में द हिन्दु की कवरेज सबसे बेहतर है, इसकी खबरों में गहराई होती है। 
एक दिन में लगभग 120 खबरें प्रकाशित होती है इस हिसाब से 30 दिन में 3600 खबरें। इनमें औसतन 300 खबरें ही विकास से जुड़ी होती है इससे यह साफ जाहिर होता है कि अब भी विकासात्मक खबरें 10 प्रतिशत से भी कम है। अब धीरे -धीरे इसका औसत बढ़ रहा है। जो विकासात्मक पत्रकारिता के लिए अच्छी बात है।

अन्तःवस्तु विष्लेषण - पांच पत्रिकाओं का सर्वेक्षण

\begin{tabular}{|l|l|}
\hline पत्रिकाएं & विकास संबंधी खबरें \\
\hline योजना & $90 \%$ \\
\hline इंडिया टुडे & $14 \%$ \\
\hline आउटलुक & $15 \%$ \\
\hline अहा! जिंदगी & $12 \%$ \\
\hline प्रथम प्रवक्ता & $10 \%$ \\
\hline
\end{tabular}

समयावधि- जनवरी 2011 से दिसम्बर 2012

\section{योजना}

विकास को समर्पित इस पत्रिका में मुख्यतः विकास संबंधि खबरें, लेख आदि का प्रकाशन किया जाता है। हर महीने किसी खास मुद्दे विस्तृत जानकारी देने की कोशिश की जाती है। बालश्रम, जलसंरक्षण, खेती के नई तकनीक, पशुपालन, सिंचाई व्यवस्था आदि मुद्दों पर साल 2011 में विशेष जानकारी दिया गया।

\section{अहा!जिंदगी}

सकारात्मक खबरें देने वाली यह पत्रिका वर्तमान में लोगों द्वारा काफी पसंद की जा रही है। इसमें विकास संबंधी प्योर खबरें तो कम ही होती है परन्तु कई खबरें और लेख विकास से जुड़े होते है। जीवन मूल्य से जुड़ी कई खबरें पाठकों को विकास के लिए प्रोत्साहित करती है।
आउटलुक

राजनीति, कला साहित्य, पर्यटन, फिल्म आदि सभी क्षेत्रों का कवरेज करने का प्रयास इस प्रतिका में किया जाता है। महीने में दो बार प्रकाशित होने वाली इस पत्रिका में दो-तीन खबरें विकास से जुड़ी होती है।

इंडिया टुडे

मुख्य रुप से यह राजनीतिक पत्रिका है राजनीतिक विकास को इसमें प्रमुखता से स्थान दिया जाता है । ग्रामीण विकास की भी कई खबरें व लेख इसमें प्रकाशित की जाती है। जैसे- एक एक बूंद से घड़ा भरे, अधूरा रहा आदर्श ग्राम का सपना।

\section{प्रथम प्रवक्ता}

स्कूल है तो पढ़ाई नहीं, चले पांच साल पहुंचे आधा कोष, इस तरह विकास के कुछ मुद्दे का प्रकाशन नियमित रुप से इस पत्रिका में किया जाता है। राजनीतिक पत्रकारिता से जुड़े विकास के मुद्दे पर विशेष कवरेज इसमें दी जाती है।

\section{निष्कर्ष}

अन्तःवस्तु विष्लेषण सारणी की व्याखा करने के पश्चात् शोधार्थी इस निष्कर्ष पर पहुंचा है कि विकास पत्रकारिता वर्तमान समय में बहुत ही अधिक प्रासंगिक हो गया है, क्योंकि दुनिया आज जिस मोड़ पर खड़ी है वह विकास का अन्धाधून दौर है। प्रकृति संसाधन का जबरदस्त दोहन कर सभी आगे बढ़ना चाहते है। इस अंधी दौर से बाहर निकालने में विकास पत्रकारिता पूरी तरह से सक्षम है। विकास पत्रकारिता ही विकास के नये मापदंड स्थापित करेगा।

वर्तमान उपभोगतावादी युग से नये मून्य स्थापित करने का कार्य विकास पत्रकारिता द्वारा ही संभव है यह लोगों को जागरुक करती है कि पेड़ लगाये, बिजली - पानी बचायें। गरीबी और अमीरी के बीच असामनता के खाई को कम करने में यह महत्वपुर्ण भूमिका निभा सकती है। 
विकास ही समस्त जगत का मूल आधार व उद्देश्य है विकास के बिना मनुष्य अधुरा है विकास ही शक्ति है, बल है। शक्ति और बल प्रदान करने में प्रमुख भूमिका निभाता है "विकासात्मक पत्रकारिता" । इसकी खास बात यह है कि विकास पत्रकारिता के लिए संबंधित क्षेत्र में जाना आनिवार्य है यह पत्रकारिता के वास्तविक स्वरुप को बताता है। विकास का ढॉचा कैसा होगा, आर्थिक रीढ़ कैसे सुद्दढढ होगें शिक्षण विकास पत्रकारिता से हमें मिलता है।

विकास के लिए हम नई-नई तकनीकें अपनाते हैं नई-नई तकनीकें अपना कर स्वयं लाभान्वित होते है तथा समाज, राष्ट्र एवं विश्व को भी लाभान्वित करते हैं। लाभान्वित करनेवाली इन हस्तियों को प्रकाश में लाना ही विकास पत्रकारिता का अहम कार्य है। विकास पत्रकारिता हमें सिखाती है कि धटनाएँ तत्काल न घटकर, दूरगामी परिणाम पर आधारित होती है। विकास पत्रकारिता से जुड़े पत्रकार स्वभाव से खोजी तथा अन्वेषण प्रतिभा से संपन्न होते है।

लोगों के जीवन स्तर को देखकर ही वास्तविक प्रगति का अनुमान लगाया जा सकता है। जब व्यक्ति गरीबी रेखा के नीचे जीवन बसर कर रहा है तो इसे विकास नहीं कहेंगे क्योंकि जीवन स्तर बेहतर हुए बिना कोई विकास संभव नहीं। जीवन स्तर को बेहतर बनाना विकास पत्रकारिता का अभिन्न अंग है।

विकासात्मक पत्रकारिता का मूल लक्ष्य वर्गो तथा क्षेत्रों के बीच भावनात्मक एकता को बढ़ावा देना है। और तकनीकि आधारभूत ढढँचे का निर्माण कैसे होता है? समाज के आधुनिकीकरण की दिशाएँ कैसे प्रशस्त होती हैं, रुढ़ियों के त्याग की प्रेरणा कैसे प्राप्त की जाती है, स्वालंबन कैसे प्राप्त होता है इसका दिशा-निर्देश करना ही विकास पत्रकारिता का लक्ष्य है।

एक अकाट्य सत्य यह है कि भारत की एक तिहाई आबादी गरीबी रेखा के नीचे है। एक तिहाई आबादी को
दो जून का सन्तुलित भोजन भी उपलब्ध नहीं है। गरीबी का प्रश्न सही अर्थो में बेरोजगारी से जुड़ा हुआ है। दूसरे शब्दों में हम कह सकते हैं कि देश के सामने सबसे बड़ी समस्या गरीबी व बेरेजगारी है। अगर समय रहते इसका समाधान नहीं हुआ तो विकास की सारी उपलब्धियां बेकार साबित होंगी। यह समस्या जितना सरकार के लिए महत्वपूर्ण है उतना ही पत्रकारिता के लिए।

पत्रकारिता देश में व्याप्त गरीबी और बेरोजगारी के किस रूप में देखती है और जनता के समक्ष इसका विश्नेषण किस रूप में करती है। गरीबी और बेरोजगारी की समस्या के साथ सामाजिक-आर्थिक विकास के सारे महत्वपूर्ण सवाल जुड़े हुए हैं। इसका बेवाक विश्नेषण इस रूप में करना चाहिए कि जनता इसके मर्म को आसानी से समझ सके। वैसे भी जब हम विकास की बात करते हैं तो इस बात पर जोर देते हैं कि इसकी सफलता के लिए जनता की जागरूकता व विकास की प्रक्रिया में इसकी भागीदारी सबसे बड़ी शक्ति होती है जो विकास के मार्ग में सही दिशा में प्रशस्त करती है। वस्तुतः विकास की समस्याओं को राजनीतिक नारों से नहीं सुलझाया जा सकता है। इसके लिए जरूरी है कि सरकार और पत्रकारिता जगत को अधिक से अधिक संवेदनशील होना चाहिए। तभी जाकर सामाजिक-आर्थिक विकास में गुणात्मक परिवर्तन सम्भव हो सकता है। जनता की भागीदारी और सरकार की नीतियों की पारदर्शिता की समस्या का हल भी विकास पत्रकारिता के रणनीति में शामिल करना होगा। जिसके माध्यम से हम विकास के लक्ष्य को प्राप्त करने में सफल हो सकते हैं।

समाचार पत्रों में विकास संबंधी समाचारों को उतना स्थान नहीं दिया जितना विदेशी व अन्य समाचारों को दिया। जबकि भारत जैसे विकासशील देश के लिए इसकी आवश्यकता है। सामाजिक उत्थान के प्रति समाचार पत्रों की जिम्मेदारी है। लेकिन अधिकांश समाचार पत्रों का मुख्य कार्यक्षेत्र नगरों तक ही सीमित हो गया है। सम्पादक विकास पत्रकारिता से अपने आपको नहीं जोड़ पाये हैं। ग्रामीण समाज को विकास व ज्ञान की अधिक आवश्यकता है। जबकि सम्पादक इनसे 
काफी दूर बैठे हुए हैं, और उनकी दृष्टि उन पिछड़े हुए क्षेत्रों तक नहीं पहुंच पा रही है।

=

Deepak Kumar, Journalism and Mass Communication, Dev Sanskriti Vishwavidyalaya, Haridwar, India. Email Deepak.kumar@dsvv.ac.in

संदर्भ

राजकिशोर, मानव अधिकारों का संघर्ष , 1995, वाणी प्रकाषन

डॉ. नैयर रेणुका, ग्रामीण क्षेत्र की पत्रकारिता, 2002, हरियाणा साहित्य अकादमी, पंचकुला

झुनझुनवाला भारत, आर्थिक पत्रकारिता, 2005, श्री नटराज प्रकाशन, दिल्ली

पुराणिक आलोक, आर्थिक पत्रकारिता, 2007, प्रभात प्रकाशन

पालीवाल.के.आर, 22वीं सदी का गांव, 2007, भावना प्रकाशन

कुमार भास्कार, भूमण्डलीकरण और स्री, 2008, संजय प्रकाशन, दिल्ली

डॉ. उपाध्याय कुमार अनिल, 2007, पत्रकारिता एवं विकास संचार
अवस्थी कमलेश, 2006, परंपरा और अधुनिकीकरण

नरुला उमा, डेवलपमेंट कम्युनिकेशन, 2007, हर आनंद पब्लिकेषन

सेन अमर्त्य, आर्थिक विकास और स्वातंत्रय, 2014, राजकमल एंड सन्स

नन्द भारद्वाज, संस्कृति जनसंचार और बाजार, 2007 सामयिक प्रकाशन, नई दिल्ली

जैन रमेश, पत्रकारिता राजस्थान प्रकाशन, 1995, राजस्थान प्रकाशन, जयपुर

उपाध्याय अनिल, मास मीडिया एवं विकास के आयाम, 2007, विश्वविद्यालय प्रकाशन 